\title{
Application of the SBA method to solving optimal control problem governed by systems of nonlinear partial differential equations
}

\author{
Bakari Abbo $^{1}$, Pare Youssouf ${ }^{2 *}$, Bassono Francis ${ }^{2}$, Marayi Choroma ${ }^{1}$, Blaise Some $^{2}$ \\ ${ }^{1}$ University of $N$ 'djamena \\ ${ }^{2}$ University of Ouagadougou \\ *Corresponding author E-mail: pareyoussouf@yahoo.fr
}

\begin{abstract}
In this article, we are interested in solving optimal control problems governed by a system of nonlinear partial differential equations by the iterative method SBA(combination of the Adomian method, successive approximations method and the principle of Picard) [17]. This technique allows reducing the problem of optimal control of the error function

in a classical minimization problem.

$$
J=\int_{0}^{T}(u(l, t)-\varepsilon)^{2} d t
$$
\end{abstract}

Keywords: Adomian method, successive approximations method, Picard principle, SBA method.

\section{Introduction}

We are interested in the resolution of optimal control problems governed by systems of nonlinear partial differential equations of Cauchy type:

$$
(\mathrm{S}):\left\{\begin{array}{l}
u_{t}=F(t) L(u, v)+F(t) G(t) N(u, v)+\varphi(u, v) ; 0<t<T, 0<x<L \\
v_{t}=G(t) \tilde{L}(u, v)+F(t) G(t) \tilde{N}(u, v)+\psi(u, v) ; 0<t<T, 0<x<L \\
u(0)=f \\
v(0)=\tilde{f}
\end{array}\right.
$$

Modeling many phenomena $[13,16]$ in biomathematics. This is for instance the case of two substances of respective concentrations $u(t, x)$ and $v(t, x)$ in interaction; and $\psi$ can represent different types of nonlinearity.

The problem is not the classical resolution of the system (S), it comes to determine the control functions

$$
F, G:[0 ; T] \rightarrow \mathbb{R}
$$

Minimizing the error functional

$$
J=\int_{0}^{T}(u(l, t)-\varepsilon)^{2} d t(P)
$$

Where $\mathrm{T}$ represents the observation duration of the phenomenon and $\varepsilon>0$.

Several numerical resolution methods of such systems have been developed, among others, the spectral method, the finite difference these conventional methods of resolution usually use the technique of discretization or linearization. The new technique SBA [1-5], does not discretize the system of equations and retains the biological properties of the model, and this is important for the applications [13-16].

\section{Description of the approach SBA for the system (S)}

The algorithm SBA (Some Blaise - Abbo) [1-5] is applied to the following system 


$$
(S):\left\{\begin{array}{l}
u_{t}=F(t) L(u, v)+F(t) G(t) N(u, v)+\varphi(u, v) ; 0<t<T, 0<x<L \\
v_{t}=G(t) \tilde{L}(u, v)+F(t) G(t) \tilde{N}(u, v)+\psi(u, v) ; 0<t<T, 0<x<L \\
u(0)=f \\
v(0)=\tilde{f}
\end{array}\right.
$$

In a functional space with $(L, \tilde{L})$ a pair of linear differential operator, $(N, \tilde{N})$ the nonlinear term and $u, v$ unknown functions such as

$$
u_{t}=\frac{\partial u}{\partial t}, v_{t}=\frac{\partial v}{\partial t} .
$$

Using the idea of the method of successive approximations, the above problem can be approximated by the following equivalent problem:

$$
\left(S_{\text {appr }}\right):\left\{\begin{array}{lc}
u_{t}=F(t) L\left(u^{k},{ }^{k}\right)+F(t) G(t) N\left(u^{k-1},{ }^{k-1}\right)+\varphi\left(u^{k-1},{ }^{k-1}\right) ; 0<t<T \\
v_{t}=G(t) \tilde{L}\left(u^{k},{ }^{k}\right)+F(t) G(t) \tilde{N}\left(u^{k-1},{ }^{k-1}\right)+\psi\left(u^{k-1},{ }^{k-1}\right) ; 0<t<T \\
u^{k}(0)=f, & k=1,2, \ldots \\
v^{k}(0)=\tilde{f}, & k=1,2, \ldots
\end{array}\right.
$$

Solving the problem $\left(S_{\text {appr }}\right)$ by the method of successive approximations, then we determine at each iteration $(k=1,2, \ldots)$ the following approximate solutions: $u^{1}, u^{2}, \ldots, u^{n}, \ldots, v^{1}, v^{2}, \ldots, v^{n}, \ldots$

But this requires first the choice of the initial condition $\left(u^{0}, v^{0}\right)$. the resulting solution $(u, v)$ of the problem $(S)$ sought is obtained by:

$$
(u, v)=\lim _{k \rightarrow \infty}\left(u^{k}, v^{k}\right)
$$

If $\left(u^{k}, v{ }^{k}\right)$ is convergent.

Note that various successive approximate solutions are usually obtained by using the method of Picard [17]. In the specific case, we use the decompositional method of Adomian at each iteration step in choosing a hypothesis $\left(u^{0}, v^{0}\right)$. Reconsidering the following problem:

$$
\left(S_{\text {appr }}\right):\left\{\begin{array}{lc}
u_{t}=F(t) L\left(u^{k},{ }^{k}\right)+F(t) G(t) N\left(u^{k-1},{ }^{k-1}\right)+\varphi\left(u^{k-1},{ }^{k-1}\right) ; 0<t<T \\
v_{t}=G(t) \tilde{L}\left(u^{k}, v^{k}\right)+F(t) G(t) \tilde{N}\left(u^{k-1,},{ }^{k-1}\right)+\psi\left(u^{k-1, v}{ }^{k-1}\right) ; 0<t<T \\
u^{k}(0)=f, & k=1,2, \ldots \\
v^{k}(0)=\tilde{f}, & k=1,2, \ldots
\end{array}\right.
$$

We use the Adomian method to solve the problem at each iteration step. But above all, we note that after the choice of the couple $\left(u^{0}, v^{0}\right)$,

And $\left(g_{k}, \tilde{g}_{k}\right)$ is a pair of functions depending only on $t$ (in the case of ODE) or on $x$ and $t$ (in the PDE).

As a result the problem $\left(S_{\text {appr }}\right)$ becomes

$$
\left\{\begin{array}{lr}
u_{t}^{k}=L\left(u^{k}, v^{k}\right)+g_{k}, 0<t<T \\
v_{t}^{k}=\tilde{L}\left(u^{k}, v^{k}\right)+\tilde{g}_{k}, 0<t<T \\
u^{k}(0)=f \quad k=1,2, \ldots \\
v^{k}(0)=\tilde{f} \quad k=1,2, \ldots
\end{array}\right.
$$

Which is a problem of semi-linear easily solved by the Adomian method EDO. 
Indeed, witch the choice of operators

$$
L_{1}=\frac{\partial}{\partial t} \text { And } L_{1}^{-1}=\int_{0}^{t}(.) d s
$$

The system of equations

$$
\left\{\begin{array}{l}
u_{t}^{k}=L\left(u^{k}, v^{k}\right)+N\left(u^{k-1}, v^{k-1}\right)+\varphi\left(u^{k-1}, v^{k-1}\right) \\
v_{t}^{k}=\tilde{L}\left(u^{k}, v^{k}\right)+\tilde{N}\left(u^{k-1}, v^{k-1}\right)+\psi\left(u^{k-1}, v^{k-1}\right)
\end{array}\right.
$$

Can be rewritten as follows:

$$
\left\{\begin{array}{l}
u^{k}=u^{k}(0)+L_{1}^{-1}\left(L\left(u^{k}, v^{k}\right)\right)+L_{1}^{-1}\left(N\left(u^{k-1}, v^{k-1}\right)\right)+L_{1}^{-1}\left(\varphi\left(u^{k-1}, v^{k-1}\right)\right) \\
v^{k}=v^{k}(0)+L_{1}^{-1}\left(\tilde{L}\left(u^{k}, v^{k}\right)\right)+L_{1}^{-1}\left(\tilde{N}\left(u^{k-1}, v^{k-1}\right)\right)+L_{1}^{-1}\left(\psi\left(u^{k-1}, v^{k-1}\right)\right)
\end{array}\right.
$$

Which is a canonical form of Adomian. And we can deduce the following Adomian algorithm:

$$
\left\{\begin{array}{lrl}
u_{0}^{k}=u^{k}(0)+L_{1}^{-1} g_{k}, & k=1,2, \ldots \\
u_{n}^{k}=L_{1}^{-1}\left(L\left(u_{n-1}^{k}, v_{n-1}^{k}\right)\right)+L_{1}^{-1}\left(\varphi\left(u^{k-1}, v^{k-1}\right)\right), & k=1,2, \ldots \\
v_{0}^{k}=v^{k}(0)+L_{1}^{-1} \tilde{g}_{k}, & k=1,2, \ldots \\
v_{n}^{k}=L_{1}^{-1}\left(L\left(u_{n-1}^{k}, v_{n-1}^{k}\right)\right)+L_{1}^{-1}\left(\psi\left(u^{k-1}, v^{k-1}\right)\right), & k=1,2, \ldots
\end{array}\right.
$$

Then the solution at each step is given by:

$$
\left(u^{k},{ }^{k}\right)=\left(\sum_{n=0}^{\infty} u_{n}^{k}, \sum_{n=0}^{\infty} v_{n}^{k}\right), \quad k=1,2, \ldots
$$

And the solution $(u, v)$ of $(S)$ is obtained by: $(u, v)=\lim _{k \rightarrow \infty}\left(u^{k}, v^{k}\right)=\Lambda(F(t) ; G(t))$. This solution typically solve the problem $(P)$. Indeed, with the analytical solution obtained by the SBA method, the functional $J$ can be rewritten as follows: $J=\int_{0}^{T}\left(\Lambda_{1}(F(t), G(t))-\varepsilon\right)^{2} d t(P)$

Where we replace by its equivalent: $u(l)=\Lambda_{1}(F) t(\phi)$ Let's put $\Lambda_{2}(m, t)=(F(t), G(t))$ and $J$ becomes

$$
J=\int_{0}^{T}\left(\Lambda_{1} \Lambda_{2}(m, t)-\varepsilon\right)^{2} d t \quad(P) .
$$

Thus we get a classical minimization problem. Thereby minimizing the error functional $J$ with respect to $F(t), G(t)$ is equivalent to minimizing $J$ relative to the constant parameter $m$.

\section{Numerical examples}

\subsection{Application to systems of nonlinear partial differential equations}

We are intending to determine the control functions $F(t)$ and $G(t)$ which minimize the functional error

$$
J=\int_{0}^{T}(u(l, t)-\varepsilon)^{2} d t(P)
$$

Where $u(t, x)$ verifies the system of nonlinear partial differential equations following: 


$$
(S):\left\{\begin{array}{l}
u_{t}=F(t) L(u, v)+F(t) G(t) N(u, v)+\varphi(u, v) ; 0<t<T \\
v_{t}=G(t) \tilde{L}(u, v)+F(t) G(t) \tilde{N}(u, v)+\psi(u, v) ; 0<t<T \\
u(0)=f \\
v(0)=\tilde{f}
\end{array}\right.
$$

Where $\mathrm{T}$ represents the observation duration of the phenomenon and $\varepsilon>0$. in the various examples below we take $F(t)=G(t)=e^{m t}, m$ is a constant.

\section{Example 1:}

Considering the nonlinear partial differential equations as follow:

Which solution is?

$$
(p):\left\{\begin{array}{l}
\frac{\partial u}{\partial t}=e^{m t} \frac{\partial^{2} v}{\partial x^{2}}+u^{2}-v^{2}-\left(a^{2}-b^{2}\right)\left(x^{4}-\frac{4}{m^{2}}\left(e^{m t}-1\right)^{2}\right) \\
\frac{\partial v}{\partial t}=e^{m t} \frac{\partial^{2} u}{\partial x^{2}}+u^{2}-2 u v+v^{2}-(a-b)^{2}\left(x^{2}-\frac{2}{m}\left(e^{m t}-1\right)\right)^{2} \\
u(x, 0)=a x^{2} \\
v(x, 0)=b x^{2}
\end{array}\right.
$$

$$
(u, v)=\lim _{k \rightarrow \infty}\left(u^{k}, v^{k}\right)=\left(a x^{2}+\frac{2 b}{m}\left(e^{m t}-1\right) ; b x^{2}+\frac{2 a}{m}\left(e^{m t}-1\right)\right)
$$

Obtained by the SBA algorithm [1-5].

The error functional $J$ is well

$$
J=\int_{0}^{T}\left(a l^{2}+\frac{2 b}{m}\left(e^{m t}-1\right)-\varepsilon\right)^{2} d t(P)
$$

Thereby minimizing the error functional $J$ with respect to $F(t), G(t)$ is equivalent to minimizing $J$ relative to constant parameter $m$.

The minimum of $J$ is obtained by the expression $\frac{\partial J}{\partial m}=0$ which solution is written in the following form: $m=f(a, b, l, \varepsilon, T)$, where $a, b, l, \varepsilon, T$ are real constants.

\section{Example 2:}

Considering the nonlinear partial differential equations as follow:

$$
(p):\left\{\begin{array}{l}
\frac{\partial u}{\partial t}=e^{m t} \frac{\partial^{2} v}{\partial x^{2}}+u^{2}-v^{2}+\cos 2 x \\
\frac{\partial v}{\partial t}=e^{m t} \frac{\partial^{2} u}{\partial x^{2}}+u+v-\sqrt{2} \cos \left(x-\frac{\pi}{4}\right) e^{\frac{1-e^{m t}}{m}} \\
u(x, 0)=\sin x \\
v(x, 0)=\cos x
\end{array}\right.
$$

Which solution is?

$$
(u, v)=\lim _{k \rightarrow \infty}\left(u^{k},{ }^{k}\right)=\left(\sin x \cdot \operatorname{ch}\left(\frac{e^{m t}-1}{m}\right)-\cos x \cdot \operatorname{sh}\left(\frac{e^{m t}-1}{m}\right) ; \cos x \cdot \operatorname{ch}\left(\frac{e^{m t}-1}{m}\right)-\sin x \cdot \operatorname{sh}\left(\frac{e^{m t}-1}{m}\right)\right)
$$

Obtained by the SBA algorithm, we can determine in the same manner as example1, the controls functions $F(t), G(t)$. The functional error $J$ is: 


$$
J=\int_{0}^{T}\left(\sin l \cdot c h\left(\frac{e^{m t}-1}{m}\right)-\cos l \cdot s h\left(\frac{e^{m t}-1}{m}\right)-\varepsilon\right)^{2} d t \quad(P)
$$

Thereby minimizing the functional error $J$ with respect to $F(t), G(t)$ is equivalent to minimizing $J$ relative to constant parameter $m$.

The minimum of $J$ is obtained by the expression $\frac{\partial J}{\partial m}=0$ which solution is written in the following form: $m=f(a, b, l, \varepsilon, T)$, where $a, b, l, \varepsilon, T$ are real constants.

\section{Discussion}

The above examples illustrate the effectiveness of the SBA method in solving optimal control problems. Unlike the methods mentioned above, the SBA algorithm reduces the resolution of an issue of control, problem solving classical minimization. This is of great interest to users.

The SBA method can contribute effectively to solving not only the optimal control, but also the control in general.

\section{Conclusion}

This work allowed us to show that the SBA method can contribute to solving optimal control problems in one dimension space EDP nonlinear Cauchy-type systems.

\section{References}

[1] Bakari Abbo, « Nouvel algorithme numérique de résolution des équations différentielles ordinaires (EDO) et les aux dérivées partielles non linéaires ». Thèse de Doctorat unique de l'Université de Ouagadougou (Janvier 2007)

[2] Bakari Abbo, Abba Danna, Pare Youssouf and Blaise Some, "Extension of the new iterative approach of Adomian algorithm to partial differential equations(PDE) strongly nonlinear with initial and boundary conditions”. Far East Journal of Mathematical Sciences (FJMS) Volume 75, Number 2, 2013, Pp 245-255.

[3] Youssouf Pare, Abbo Bakari, Rasmane Yaro and Blaise some, « Solving first kind Abel integral equations using the SBA numerical method ». Nonl. Analysis and Differential Equations, Vol. 1, 2013, no.3, pp 115-128.

[4] Pare Youssouf, Bassono Francis, Bakari Abbo and Blaise some,"Generalisation of SBA (SOME BLAISE-ABBO) algotithm for solving Cauchy nonlinear PDE (partial differential nonlinear equation) in $\mathrm{n}$ dimension space". Advances in Differential Equations and Control Processes. Vol 4, Num 2, 2009, pp 79-94.

[5] Pare Youssouf, Saley Bisso, Some Blaise, Ousseini So and Bakari Abbo. « A numerical method for solving Cauchy evolution problem of partial differential equations (PDEs) for several variables, Internat.J.Numer.Methods Appl. Vol 1(1) 2009, pp 87-100.

[6] B.Abbo, N.Ngarasta, B. Mampassi, B. Some and L. Some, "A New Approach of the Adomian Algorithm for Solving Nonlinear Ordinary or Partial Differential Equations”. Far East J. Appl. Math. 23(3) 2006, pp299-312.

[7] Pare Youssouf: «Résolution de quelques équations fonctionnelles per la méthode SBA (Somé Blaise-Abbo) ». Thèse de Doctorat unique de l’Université de Ouagadougou, Mai 2010.

[8] Bassono.Francis, «Résolution Numérique comparée de quelques équations fonctionnelles de quelques équations fonctionnelles par les méthodes: SBA, décompositionnelle d'Adomian ET perturbations ». Thèse Doctorat unique Université de Ouagadougou (Janvier 2013).

[9] A.Wazwaz, « The numerical solution of sixth-order boundary value problems by the modified decomposition method ». Applied Mathematics and computation 118 (2001) 311-325.

[10] Andreas Ruffing \& al. "Comparing Algebraic and Numerical Solutions of Classical Diffusion Process Equations in Computation Financial Mathematics “. Discrete Dynamics in Nature and Society, Vol 6, pp. 157-169.

[11] Blaise some, «Convergence of the Adomian method applied to Fredholm integro-differential equations ». Africa Mat. 3, 14 (2001), pp71-88.

[12] K. ABBAOUI, «Les fondements de la méthode décompositionnelle d'Adomian et application a la résolution de problèmes issus de la biologie et de la médicine ». Thèse de doctorat de l'Université Paris VI. Octobre 1995.

[13] K. ABBAOUI and Y. CHERRUAULT, "The Decomposition method applied to the Cauchy problem". Kybernetes, (28, 1), pp68-74, 1999.

[14] K. ABBAOUI and Y. CHERRUAULT, "Convergence of Adomian method applied to differential equations". Math. Comput. Modelling (28, 5), pp 103-109, 1994.

[15] G.Adomian, "Nonlinear Stochastic Systems Theory and Application to physic", Kluwer Academic Pub (1989).

[16] Y. CHERRUAULT, "Modèles et Méthodes Mathématiques pour les Sciences du Vivant”.Presses universitaires de France; Paris (1998).

[17] Hassan K.Khalil, "Nonlinear Systems" second edition. Prentice-Hall, Inc 1996.

[18] B. Mampassi, B. Saley and Some Blaise, "Solving some Nonlinear Reaction-diffusion equations using the new Adomian Decomposition Method" ADJM, Vol 1, no1, pp1-9(2003).

[19] Murray J.D, "Mathematical Biology" Springer, Berlin(1989)

[20] Murray J.D, "Nonlinear Differential Equation Models in Biology" Clarendon Press; Oxford, 1977.

[21] Shepley L. Ross, "Introduction to Ordinary Differential Equations" University of new Hampshire, second edition(1974)

[22] T. Mavoungou \& Y. Cherruault, "Numerical Study of Fisher's Equation by Adomian's Method" Mathl. Comput. Modelling Vol.19. No1, pp. 89-95, 1994. 